\title{
Estado de salud de los muy ancianos: situación clínica y funcional de la población centenaria
}

\author{
R. RABUÑAL REY, R. MONTE SECADES, M. D. VEIGA CANDO', M. T. RIGUEIRO \\ VELOSO, M. J. LÓPEZ DÍAZ, E. J. CASARIEGO VALES, J. GUERRERO \\ LOMBARDÍA
}

Servicio de Medicina Interna. ${ }^{1}$ Unidad de Hospitalización a Domicilio. Complexo Hospitalario Xeral-Calde. Lugo HEALTH STATUS OF THE OLDEST OLD: FUNCTIONAL AND
MEDICAL SITUATION IN CENTENARIANS

\section{RESUMEN}

Objetivo: Estudio prospectivo y descriptivo de la población centenaria del área sanitaria de Lugo, valorando su situación social, médica y funcional y la relación de ésta con los parámetros analíticos básicos.

Material y métodos: Entre enero de 2001 y septiembre de 2003 se realizó una visita domiciliaria cumplimentandose un protocolo que incluía situación social, historia clínica, exploración física, estado funcional (Índice de Barthel -IB-) y extracción de muestras de sangre.

Resultados: Se entrevistó a 54 centenarios, 16 varones y 38 mujeres. El 75,9\% eran viudos; el $87 \%$ viven con su familia, en núcleos urbanos (57,4\%); el 79,6\% cursó estudios. Todos tienen ingresos propios. En sus antecedentes médicos destaca que el $64,8 \%$ presenta alteraciones de vision o audición, el 81,5\% consume medicamentos, el 59,3\% sufrío intervenciones quirúrgicas y el $46,3 \%$ ingresó por causas medicas. La cobertura vacunal es baja. La valoración funcional, mediante el IB, obtuvo una puntuación media de $59 \pm 36,4$, mostrando una diferencia significativa $p<0.003$ entre varones $(82,7 \pm 28,7)$ y mujeres $(49,6 \pm 35,1)$. Se extrajeron muestras de sangre a 51 centenarios, no demostrándose diferencias significativas entre sexos ni tampoco según el nivel de dependencia.

Conclusión: La población centenaria del área sanitaria de Lugo es similar a la descrita en otros países. Es un grupo heterogéneo, con predominio de mujeres, pero su situación clínica y funcional es significativamente peor que la de los varones. No hemos encontrado relación entre los parámetros hematológicos y nutricionales y el nivel de dependencia funcional de los centenarios.

PALABRAS CLAVE: Centenario. Activities of Daily Living. Longevidad.

\section{ABSTRACT}

Objective: We planned a prospective and descriptive analysis of the centenarian population in Lugo sanitary area, establishing their social, functional and medical status as well as its relationship with their basic hematological and biochemical parameters.

Material and methods: During the study period between january 2001 and september 2003, participants were visited at home by a doctor and a nurse. The following variables were assessed: social status, past medical history, physical examination, functional status (Barthel index), blood analysis.

Results: 54 centenarians were interviewed, 16 men and 38 women. $75,9 \%$ were widows; $87 \%$ lived with their family and $57,4 \%$ in urban areas. 79,6\% had followed studies. All of them had their own incomes. Regarding past medical history, 64,8\% had some visual or auditive disturbances, $81,5 \%$ were taking medical drugs, $59,3 \%$ had some surgical intervention and 46,3\% had been hospitalized for medical reasons. Their vaccination status was poor. Functional status, assessed by Barthel index, showed an average of $59 \pm 36,4$, higher in men $(82,7 \pm 28,7)$ than in women $(49,6 \pm 35,1)(p<0,003)$. Blood samples were analyzed in 51 cases, there were not significant differences among them regarding sex or physical disability.

Conclusion: The features of the centenarian population of Lugo are similar to other countries. It is an heterogeneous group. There are more women, but their clinical and functional status are significantly worse than in males. We have not founded a relationship between hematological and nutritional parameters and the degree of functional dependence in centenarians.

KEY WORDS: Centenarians. Activities of Daily Living. Longevity.

Rabuñal Rey R, Monte Secades R, Veiga Cando MD, Rigueiro Veloso MT, López Díaz MJ, Casariego Vales EJ, Guerrero Lombardía J. Estado de salud de los muy ancianos: situación clínica y funcional de la población centenaria. An Med Interna (Madrid) 2004; 21 : $543-547$.

\section{INTRODUCCIÓN}

Con el aumento del nivel de vida y la mejora de los sistemas de salud no es raro alcanzar edades avanzadas, por lo que atender a pacientes centenarios, aunque no es habitual en nuestros hospitales, ha dejado de ser un hecho anecdótico. Asimismo las previsiones indican un aumento progresivo de esta población. En Galicia en 1998 un 19,3\% de la población era mayor de 65 años (de entre ellos 359 centenarios), incrementandose en la provincia de Lugo hasta el 25,67\% (65 centenarios) (1). Por otra parte, la Comisión de Población y Desarrollo de las Naciones Unidas prevé que el número de centenarios a nivel mundial pasará de 155.000 en el año 2000 a 2.189.000 en el 2050 (2). 
El estudio de los centenarios nos permite, por una parte, analizar los factores relacionados con la longevidad, y por otra, conocer su estado de salud, con el fin de establecer los programas necesarios para conseguir la máxima independencia funcional. Actualmente existen varios grupos de investigadores que mantienen registros de centenarios (3-6), en su mayoría orientados desde una perspectiva demográfica. En España son escasos los estudios publicados sobre este tema, enfocados desde un punto de vista psicosocial (7) o sobre pacientes hospitalizados $(8,9)$.

El objetivo del presente estudio es realizar de forma prospectiva un análisis descriptivo de la situación de la población centenaria del área sanitaria de Lugo, valorando su situación social, clínica y funcional.

\section{MÉTODO}

Estudio prospectivo que describe las características de las personas de 100 años o más que residen en el Área Sanitaria de Lugo, realizado entre enero de 2001 y septiembre de 2003.

La identificación de los casos se realizó recogiendo de la Tarjeta Individual Sanitaria (SERGAS) los datos correspondientes a todos los mayores de 100 años del área. Tras solicitar autorización telefónica al paciente o al cuidador responsable, se realizó una visita domiciliaria por parte de un médico y una enfermera. Se cumplimentó un protocolo que incluía datos de filiación, situación social, historia clínica, exploración física (constantes vitales, peso, talla, auscultación cardiopulmonar, inspección en busca de edema o úlceras de presión, y exploración neurológica y de los órganos de los sentidos) y estado funcional medido mediante el Indice de Barthel (IB) (10). Asimismo se extrajeron muestras de sangre para determinación de parámetros hematológicos y bioquímicos básicos (hemograma, parámetros nutricionales, función renal y hepática, TSH, vitamina $B_{12}$ y ácido fólico). Posteriormente se envió a cada sujeto una copia del resultado de sus análisis indicándoles, en caso necesario, la conveniencia de ponerse en contacto con su médico de familia.

Los datos obtenidos se incluyeron en una base de datos creada en Access. En el análisis estadístico se utilizó la técnica Chi cuadrado con la corrección de Yates cuando fue preciso, en la comparación de las variables cualitativas. En la comparación de dos variables cuantitativas se utilizo el test $\mathrm{T}$ de Student, previa comprobación de normalidad. El nivel de significación estadística fue $\mathrm{p}<0,05$. En el análisis se utilizó el paquete estadístico SPSS 8.01.

\section{RESULTADOS}

De entre los 236.063 habitantes del área sanitaria de Lugo se contactó con 58 centenarios ( $0,02 \%$ de la población), de los cuales 54 (16 varones y 38 mujeres) concedieron autorización para la visita y 51 accedieron a la extracción de muestras sanguíneas. En la tabla I se exponen sus edades, parámetros antropométricos y situación funcional.

La mayoría de los centenarios viven con su familia (87\%). Más de la mitad $(57,4 \%)$ residen en núcleos urbanos, siendo en general las condiciones de la vivienda aceptables; todas disponen de agua corriente, electricidad y baño completo, aunque sólo el 64,8\% dispone de calefacción central; el 96,3\%

\section{TABLA I}

CARACTERÍSTICAS DE LOS CENTENARIOS ESTUDIADOS

\begin{tabular}{lccc}
\hline & Varones & Mujeres & Total \\
\hline Número & 16 & 38 & 54 \\
Edad & $100,6(1,1)$ & $100,9(1,4)$ & $100,9(1,3)$ \\
Talla & $164,4(10,4)$ & $151(8)$ & $155,8(10,4)$ \\
Peso & $65,2(13)$ & $54,5(13,1)$ & $57,7(13,9)$ \\
IMC & $23,9(3,5)$ & $23,8(5)$ & $23,8(4,7)$ \\
TAS & $132,9(15,4)$ & $127,7(20,3)$ & $129,3(19,9)$ \\
FC & $69,9(9,8)$ & $76,5(12,3)$ & $74,5(11,9)$ \\
Índice de Barthel & $82,7(28,7)$ & $49,6(35,1)$ & $59(36,4)$ \\
\hline
\end{tabular}

Valores expresados como media (DE)

tiene escaleras, pero únicamente un $27,8 \%$ de los hogares tienen ascensor. Todos los centenarios perciben algún tipo de ingreso, en su mayoría pensión contributiva (63\%). El 79,6\% cursó estudios, aunque 11 (20,4\%, 10 mujeres y 1 varón) se reconocen analfabetos. En cuanto al estado civil 5 estaban casados, 8 solteros y el resto $(41,75,9 \%)$ eran viudos, sin diferencias entre sexos. Mantenían una vida social activa 19 $(35,2 \%)$, saliendo a diario de su domicilio.

Entre sus antecedentes médicos destaca que el 18,5\% (10, todos varones) habían fumado al menos 20 años/paquete/día y el 22,6\% (7 varones y 5 mujeres) consumían alcohol habitualmente (entre 10 y 20 gramos de alcohol/día). El 64,8\% presentan alteraciones de visión o audición que les dificultan en gran medida su vida diaria. La frecuencia de las principales enfermedades se expone en la tabla II. Su cobertura vacunal se muestra en la tabla III, no existiendo diferencias estadísticas por sexo.

De entre las intervenciones quirúrgicas sufridas, las más frecuentes fueron extracción de catarata $(10,18,5 \%)$, herniorrafia $(7,13 \%)$, colecistectomía $(7,13 \%)$, cirugía reparadora de fractura de cadera $(5,9,3 \%)$ y prostatectomía $(4,25 \%$ de los varones). La patología médica que más frecuentemente ocasionó ingresos

\section{TABLA ॥}

FRECUENCIA DE LAS PRINCIPALES PATOLOGÍAS PRESENTES EN LOS CENTENARIOS

\begin{tabular}{lccc}
\hline & Varón & Mujer & Total \\
\hline Diabetes & $2(12,5)$ & $2(5,3)$ & $4(7,4)$ \\
HTA & 0 & $9(23,7)$ & $9(16,7)$ \\
Dislipemia & 0 & 0 & 0 \\
AVC & $2(12,5)$ & $6(15,8)$ & $8(14,8)$ \\
Demencia & $1(6,2)$ & $12(31,6)$ & $13(24,1)$ \\
Depresión & $1(6,2)$ & $5(13,2)$ & $6(11,1)$ \\
Cardiopatía & $3(18,7)$ & $12(31,6)$ & $15(27,8)$ \\
EPOC & $4(25)$ & $4(10,5)$ & $8(14,8)$ \\
Artrosis & $3(18,7)$ & $15(39,5)$ & $18(33,3)$ \\
Ulceras de presión & 0 & $5(13,2)$ & $5(9,3)$ \\
Incontinencia urinaria & $3(18,7)$ & $15(39,5)$ & $18(33,3)$ \\
Síndrome prostático & $8(50)$ & & \\
Intervenciones quirúrgicas & $11(68,7)$ & $21(55,2)$ & $32(59,3)$ \\
Ingresos médicos & $8(50)$ & $17(44,7)$ & $25(46,3)$ \\
Medicación & $11(68,7)$ & $32(84,2)$ & $43(79,6)$ \\
\hline
\end{tabular}

Valores expresados en $\mathrm{N}^{\circ}(\%)$ 


\section{TABLA III}

COBERTURA VACUNAL DE LOS CENTENARIOS

\begin{tabular}{lccc}
\hline & Varones & Mujeres & Total \\
\hline Gripe & $9(56,2)$ & $23(60,5)$ & $32(59,3)$ \\
Neumococo & $8(50)$ & $15(39,5)$ & $23(42,6)$ \\
Tétanos & $2(12,5)$ & $7(18,4)$ & $9(16,7)$ \\
\hline
\end{tabular}

Valores expresados en $\mathrm{N}^{\circ}(\%)$

previos fue la respiratoria $(8,14,8 \%)$ y cardíaca $(6,11,1 \%)$. Sólo $11(20,4 \%)$ no habian ingresado anteriormente en un hospital. El 81,5\% de los ancianos (44) consumía habitualmente algún medicamento; 20 (37\%) consumían tres o más. Los grupos farmacológicos más usados fueron los dirigidos al sistema cardiovascular (antiagregantes -11-, diuréticos -10-, inhibidores de la ECA -9- y digoxina -7-) y los sedantes del sistema nervioso central, fundamentalmente benzodiacepinas.

La valoración funcional, mediante el IB, obtuvo una puntuación media de $59 \pm 36,4$, siendo de $82,7 \pm 28,7$ para los varones y de 49,6 $\pm 35,1$ para las mujeres $(p<0,002)$. La distribución por niveles de dependencia se muestra en la tabla IV, sin diferencias significativas entre sexos; sin embargo, al agrupar a los pacientes en dependientes (IB < $60)$ e independientes (IB $\geq 60$ ) la diferencia se hace significativa $(\mathrm{p}=0,03)$.

Se practicó toma de muestras de sangre a 51 de los 54 centenarios. Se demostró anemia en 12 casos $(23,5 \%)$. Tres presentaban anemia megaloblástica por déficit de $\mathrm{B}_{12}$, uno presentaba una leucemia linfática crónica, 4 anemia en relación con insuficiencia renal crónica y los otros 4 se etiquetaron como anemia de trastorno crónico. Una paciente estaba diagnosticada de trombocitopenia idiopática. En dos pacientes $(3,9 \%)$ se detectó un componente monoclonal de IgG Kappa en relación con gammapatía monoclonal de significado incierto. En el resto de pacientes $(37,68,5 \%)$, todos los parámetros hematológicos fueron normales.

En la tabla V se muestran los parámetros hematológicos y bioquímicos básicos divididos por sexo, demostrándose unicamente diferencias significativas con respecto a los triglicéridos y prealbúmina. Se analizó asimismo la relación entre la presencia de alteraciones hematológicas y/o bioquímicas y el nivel de dependencia medido mediante el IB (Tabla VI)

\begin{tabular}{|c|c|c|c|}
\hline \multicolumn{4}{|c|}{ TABLA IV } \\
\hline \multicolumn{4}{|c|}{$\begin{array}{l}\text { VALORACIÓN FUNCIONAL SEGÚN EL ÍNDICE DE BARTHEL } \\
\qquad(P=N S)\end{array}$} \\
\hline & Varones & Mujeres & Total \\
\hline Dependencia total $(<20)$ & $1(6,2)$ & $9(23,7)$ & $10(18,5)$ \\
\hline Dependencia severa (20-35) & $1(6,2)$ & $6(15,8)$ & $7(13)$ \\
\hline Dependencia moderada (40-55) & $1(6,2)$ & $5(13,2)$ & $6(11,1)$ \\
\hline Dependencia leve $(\geq 60)$ & $13(81,2)$ & $18(47,4)$ & $31(57,4)$ \\
\hline Dependientes (IB < 60) & 3 & 20 & 23 \\
\hline Independientes (IB $\geq 60$ ) & 13 & 18 & 31 \\
\hline
\end{tabular}

Valores expresados en $\mathrm{N}^{\circ}(\%)$

\begin{tabular}{|c|c|c|c|c|}
\hline \multicolumn{5}{|c|}{ TABLA V } \\
\hline \multicolumn{5}{|c|}{$\begin{array}{l}\text { PRINCIPALES PARÁMETROS HEMATOLÓGICOS Y } \\
\text { BIOQUÍMICOS DE LOS CENTENARIOS DISTRIBUIDOS POR } \\
\text { SEXO }\end{array}$} \\
\hline & Varones & Mujeres & Total & $p$ \\
\hline Hemoglobina (g/dl) & $13,3(1,5)$ & $12,5(2)$ & $12,8(1,8)$ & ns \\
\hline Hematocrito (\%) & $39,6(4,9)$ & $37,2(6,1)$ & $38(5,8)$ & ns \\
\hline VCM (fl) & $91,8(4,8)$ & $92,1(7,2)$ & $92(6,5)$ & ns \\
\hline Leucocitos (mil/mc) & $6,7(2,6)$ & $6,1(1,7)$ & $6,3(2)$ & ns \\
\hline Plaquetas (mil/mc) & $208,9(44,1)$ & $210,1(67,9)$ & $209,8(61)$ & ns \\
\hline Fibrinogeno (mg/dl) & $444,3(76,8)$ & $404,2(78,2)$ & $417,3(79)$ & ns \\
\hline Glucosa (mg/dl) & $102,6(34,5)$ & $109,5(39)$ & $107,3(37,4)$ & ns \\
\hline Urea (mg/dl) & $63,6(20,4)$ & $61(37,6)$ & $61,9(32,9)$ & ns \\
\hline Creatinina (mg/dl) & $1,3(0,4)$ & $1,2(0,4)$ & $1,3(0,4) \mathrm{ns}$ & \\
\hline Calcio (mg/dl) & $9(0,5)$ & $8,8(0,7)$ & $8,9(0,6)$ & ns \\
\hline Hierro ( $\mu g / d l)$ & $75,3(28,1)$ & $71(27,9)$ & $72,3(27,7)$ & ns \\
\hline Colesterol (mg/dl) & $176,4(43,5)$ & $174,4(33,7)$ & $175,7(36,6)$ & ns \\
\hline Triglicéridos (mg/dl) & $76,4(28,2)$ & $105,6(46,3)$ & $95,9(43,7)$ & 0,01 \\
\hline Proteínas (g/dl) & $6,7(0,7)$ & $6,6(0,5)$ & $6,7(0,6)$ & ns \\
\hline Albumina (g/dl) & $3,8(0,4)$ & $3,7(0,4)$ & $3,7(0,5)$ & ns \\
\hline Prealbumina (mg/dl) & $20,5(4,6)$ & $16,2(4,8)$ & $17,5(5,1)$ & 0,007 \\
\hline Transferrina (mg/dl) & $202,7(38,4)$ & $202,9(42,5)$ & $202,8(40,9)$ & ns \\
\hline Retinol (mg/dl) & $3,7(1,1)$ & $3,2(1,1)$ & $3,4(1,1)$ & ns \\
\hline Ferritina (ng/dl) & $157,9(141,5)$ & $148,4(181,8)$ & $151,3(169)$ & ns \\
\hline
\end{tabular}

Valores expresados como media (DE)

TABLA VI

PRINCIPALES PARÁMETROS HEMATOLÓGICOS Y BIOQUÍMICAS DE LOS CENTENARIOS DISTRIBUIDOS SEGÚN EL NIVEL DE DEPENDENCIA

\begin{tabular}{|c|c|c|c|}
\hline & $\begin{array}{l}\text { Dependientes } \\
\text { (IB } 0 \text { - 55) }\end{array}$ & $\begin{array}{l}\text { Independientes } \\
\quad(I B \geq 60)\end{array}$ & $p$ \\
\hline Hemoglobina (g/dl) & $12,4(2,1)$ & $12,9(1,7)$ & ns \\
\hline Hematocrito (\%) & $36,7(6,5)$ & $38,7(5,4)$ & ns \\
\hline $\operatorname{VCM}(f \mid)$ & $91,5(4,9)$ & $92,3(7,5)$ & ns \\
\hline Leucocitos (mil/mc) & $6,8(2,3)$ & $6(1,8)$ & ns \\
\hline Plaquetas (mil/mc) & $228,6(73,5)$ & $198,8(49,3)$ & ns \\
\hline Fibrinogeno (mg/dl) & $438,5(80,6)$ & $402,5(77,6)$ & ns \\
\hline Glucosa (mg/dl) & $105,1(23,8)$ & $105,1(40)$ & ns \\
\hline Urea (mg/dl) & $66,9(34,5)$ & $58,9(32,5)$ & ns \\
\hline Creatinina (mg/dl) & $1,3(0,5)$ & $1,2(0,4)$ & ns \\
\hline Calcio (mg/dl) & $8,8(0,6)$ & $8,9(0,6)$ & ns \\
\hline Hierro ( $\mu \mathrm{g} / \mathrm{dl})$ & $62(27,9)$ & $81,1(23,4)$ & 0,01 \\
\hline Colesterol (mg/dl) & $170,8(42,3)$ & $178,8(33,3)$ & ns \\
\hline Triglicéridos (mg/dl) & $110,7(51,8)$ & $87,9(44,6)$ & 0,05 \\
\hline Proteínas (g/dl) & $6,6(0,5)$ & $6,7(0,7))$ & ns \\
\hline Albúmina (g/dl) & $3,5(0,4)$ & $3,9(0,4)$ & 0,005 \\
\hline Prealbumina (mg/dl) & $16,5(4,7)$ & $18,1(5,4)$ & ns \\
\hline Transferrina (mg/dl) & $198,2(48,4)$ & $205,1(36,7)$ & ns \\
\hline Retinol (mg/dl) & $3,4(0,9)$ & $3,4(1,3)$ & ns \\
\hline Ferritina (ng/dl) & $204,5(232,8)$ & $111,1(99,9)$ & 0,05 \\
\hline
\end{tabular}

Valores expresados como media (DE) 
demostrándose que los dependientes presentan cifras de albúmina más bajas y de ferritina más altas que los funcionalmente independientes.

\section{DISCUSIÓN}

El propósito de este estudio es determinar el estado de salud de las personas que llegan a centenarios, con el fin de conocer cómo es esta población, si es comparable a la analizada en otros países, y qué medidas pueden adoptarse para mejorar su situación funcional.

Un aspecto relevante del presente estudio es que a diferencia de otros previos realizados en nuestro país $(8,9)$, este es prospectivo y realizado en el domicilio del paciente, en situación basal, no en relación con una consulta u hospitalización. Además el $93 \%$ de los sujetos localizados aceptaron participar en el estudio, lo cual reduce posibles sesgos de selección. Un posible sesgo vendría dado por errores en la fecha de nacimiento en la tarjeta sanitaria, aunque estimamos que éste es bajo, ya que el hecho contrario, personas más jóvenes etiquetadas como centenarias, sólo se produjo en 4 casos.

En cuanto a los aspectos sociales se aprecian pocas diferencias respecto a lo publicado previamente en nuestro medio (7). La mayoría son viudos, viven con su familia, han cursado únicamente estudios primarios, y tienen ingresos propios; en cambio ha crecido el porcentaje de centenarios residentes en áreas urbanas, con la consiguiente mejora en las condiciones de la vivienda, lo cual debe estar en relación con el progresivo desplazamiento de población de zona rural a urbana.

Al igual que en otros estudios (11-18), las mujeres centenarias sobrepasan a los varones en un índice superior a 2:1; se han propuesto diversas hipótesis para explicar este hallazgo, desde un supuesto efecto protector del segundo cromosoma $\mathrm{X}$ hasta la eliminación de toxinas mediante las perdidas menstruales, aunque probablemente la mayor frecuencia en el varón de habitos de vida desfavorables (alcohol, tabaco) y sus patologías asociadas, acompañado de factores sociales como la práctica por parte de los hombres de profesiones de riesgo, su mayor participación en conflictos bélicos y en actividades potencialmente peligrosas como la conducción, bastarían para explicar estas diferencias (19).

Tanto hombres como mujeres tienen importantes antecedentes quirúrgicos, no siendo las intervenciones distintas de las realizadas en otros grupos de muy ancianos $(20,21)$. El elevado uso de medicamentos también ha sido descrito en otros estudios $(12,14)$, sin diferencias entre los grupos farmacológicos más usados. De entre los antecedentes médicos destaca el bajo porcentaje de factores de riesgo vascular (diabetes, hipertensión, dislipemia) que podría estar en relación con un sesgo de supervivencia, debido al fallecimiento precoz de la población que sí presenta esos factores de riesgo. A pesar de todos estos antecedentes médicos, los centenarios tienen una escasa cobertura vacunal, hecho ya descrito en otros grupos de longevos (22).

La prevalencia registrada de antecedentes medicoquirúrgi- cos apoya la idea de que los centenarios no son personas con una salud excepcional en relación con una carga genética ideal, sino que han ido sorteando las diversas vicisitudes que se les plantearon hasta llegar a esa avanzada edad. Futuros estudios en el campo de la genómica deberán aclarar este punto (23).

La prevalencia de demencia, aunque alta sobre todo en las mujeres, es menor de la descrita en otras series (24-26). Este hecho podría estar en relación con un sesgo de selección, ya que la mayoría de las series están basadas en pacientes institucionalizados, y presentan un elevado porcentaje de negativas a participar en los estudios, al contrario que la nuestra en la que los pacientes residen predominantemente con su familia y el número de negativas a participar en él fue bajo.

Se analizó la capacidad funcional mediante el IB. Encontramos, al igual que otros grupos $(11,14,16)$, que más de la mitad de nuestros centenarios son relativamente independientes, con mayor grado de dependencia en las mujeres centenarias. Aunque no está clara la causa de estas diferencias, se ha sugerido que la mayor masa muscular de los varones hace que tengan más facilidad en conservar las habilidades que miden las escalas de actividades de la vida diaria (27).

Las alteraciones hematológicas encontradas concuerdan con las que se presentan con mayor frecuencia en los ancianos $(28,29)$. El 68,5\% de los centenarios no presenta alteraciones en el hemograma, lo cual avala la hipótesis de que la anemia en el anciano no es una característica más de la vejez, sino que es signo de patología subyacente (30). Asimismo no hay alteraciones reseñables en los parámetros bioquímicos estudiados. Tampoco encontramos grandes diferencias al analizar los resultados por sexo, ni según el grado de dependencia, en contra de lo que sugieren algunos estudios (31). De todas formas si analizamos los resultados en detalle observamos que los centenarios con peor estado funcional tienen cifras más elevadas de plaquetas, ferritina y fibrinógeno respecto a los independientes funcionalmente; también vemos que presentan valores más bajos de hemoglobina, albúmina, prealbúmina y transferrina. Estos resultados, aunque no son estadísticamente significativos, quizás por el pequeño tamaño de la muestra y la elevada dispersión de algunos parámetros, parecen sugerir que el peor estado funcional puede estar relacionado con inflamación crónica y desnutrición. Posteriores estudios deben confirmar esta hipótesis.

En resumen, la población centenaria del área sanitaria de Lugo es similar a la descrita en otros países. Es un grupo heterogéneo, en el que es más frecuente encontrar mujeres centenarias, pero su situación clínica y funcional es significativamente peor que la de los varones de igual edad. Aunque no hemos encontrado relación entre los parámetros hematológi$\cos$ y nutricionales y el nivel de dependencia funcional de los centenarios, los centenarios más independientes parecen tener mejor estado nutricional y ausencia de parámetros inflamatorios en su analítica.

Con el fin de caracterizar completamente este grupo de población sería conveniente establecer un registro centralizado, al igual que sucede en otros países, que permitiera incrementar significativamente el número de centenarios estudiados. 


\section{Bibliografía}

1. Plan galego de persoas mayores 2001-2006. Conselleria de Sanidade e Servicios Sociais. Xunta de Galicia. 2001.

2. Programme implementation and future programme of work of the Secretariat in the field of population. Consultado el 16-8-2003. Disponible en: http://www.un.org/documents/ecosoc/cn9/2000/ecn92000-6.htm

3. The Georgia Centenarian Study. Consultado el 16-8-2003. Disponible en: http://www.geron.uga.edu/centenarian_study.html

4. The Swedish Centenarian Study. Consultado el 16-8-2003. Disponible en: http://www.geron.lu.se/centena1.htm

5. The New England Centenarian Study. Consultado el 16-8-2003. Disponible en: http://www.bumc.bu.edu/Departments/HomeMain.asp? DepartmentID $=361$

6. The Okinawa Centenarian Study. Consultado el 16-8-2003. Disponible en: http://okinawaprogram.com/

7. Fraiz Calvo X. A poboación centenaria de Galicia. Colección Servicios Sociais, Serie Monografías, $n^{\circ}$ 6. Universidade de Santiago de Compostela, 1993.

8. Rabuñal Rey R, Monte Secades R, Rigueiro Veloso MT, Casariego Vales EJ, Ibáñez Alonso MD, García Pais MJ. Pacientes centenarios atendidos en un hospital general. Rev Clin Esp 2002; 202 (6):326-8

9. Martín García A, Ríos Luna A, Fahandezh-Saddi Díaz H, Martínez Gómiz JM, Villa García A, Rodríguez Álvarez J: Fractura de cadera en pacientes centenarios. Rev Ortop Traumatol 2003; 47: 101-106.

10. Mahoney FI, Barthel DW. Functional evaluation: the Barthel Index. Md State Med J 1965; 14: 61-65.

11. Allard M, Robine JM. Les centenaires français. Etude de la fondation IPSEN. Serdi Editores. Paris, 2000.

12. Kinzel T, Wekstein D, Kirkpatrick C. A social and clinical evaluation of centenarians. Exp Aging Res 1986; 12: 173-6.

13. Perls TT, Morris JN, Ooi WL, Lipsitz LA. The relationship between age, gender and cognitive performance in the very old: the effect of selective survival. J Am Geriatric Soc 1993; 41: 1193-201.

14. Andersen-Ramberg K, Schroll M, Sci M, Jeune B. Healthy centenarians do not exist, but autonomous centenarians do: a population-based study of morbidity among Danish centenarians. J Am Geriatr Soc 2001; 49: 900-908.

15. Ravaglia G, Forti P, Maioli F, Boschi F, Cicognani A, Bernardi M, Pratelli L, Pizzoferrato A, Porcu S, Gasbarrini G. Determinants of functional status in healthy italian nonagenarians and centenarians: a comprehensive functional assessment by the instruments of geriatric practice. $\mathrm{J}$ Am Geriatr Soc 1997; 45: 1196-1202.

16. Zeng Y, Vaupel JW, Xiao Z, Zhang C, Liu Y. The healthy longevity survey and the active life expectancy of the oldest-old in China. Popula- tion: an English selection 2001; 13: 95-116.

17. Zheng Z, Wang Z, Zhu H, Yang J, Peng H, Wang L, Li J, Jiang X, Yu Y. Survey of 160 centenarians in Shanghai. Age Ageing 1993; 22: 1619.

18. Nybo H, Gaist D, Jeune B, McGue M, Vaupel JW, Christensen K. Functional status and self-rated health in 2,262 nonagenarians: the Danish 1905 Cohort Survey. J Am Geriatr Soc 2001; 49 (5): 601-9.

19. Gjonça A, Tomassini C, Vaupel JW. Pourquoi les femmes survivent aux hommes? La Recherche 1999; 322: 70-3.

20. Fernández Lobato R, Gil López J, Marín Lucas F, Delgado Millán M, Fernández Luengas D, Martínez Santos C, Moreno Azcoitia M. Ingresos de pacientes octogenarios en un servicio de cirugía. Rev Esp Geriatr Gerontol 1999; 34: 19-24.

21. Cogbill TH, Strutt PJ, Landercasper J. Surgical procedures in centenarians. Wis Med J 1992; 91: 527-529.

22. Formiga F, López Soto A, Masanes F, Pujol R. Escasa cumplimentación de la vacunación antigripal en pacientes nonagenarios. Med Clin (Barc) 2003; 121: 117-119.

23. Perls T. Genetic and phenotypic markers among centenarians. J J Gerontol A Biol Sci Med Sci 2001; 56 (2): M67-70.

24. Powell AL. Senile dementia of extreme aging: a common disorder of centenarians. Dementia 1994; 5: 106-9.

25. Ravaglia G, Forti P, De Ronchi D, Maioli F, Nesi B, Cucinotta D, Bernardi M, Cavalli G. Prevalence and severity of dementia among northern Italian centenarians. Neurology 1999; 53: 416-8.

26. Asada T, Yamagata Z, Kinoshita T, et al: Prevalence of demencia and distribution of ApoE alleles in japanese centenarians: an almost-complete survey in Yamanashi Prefecture, Japan. J Am Geriatr Soc 1996; 44: $151-5$.

27. Andersen-Ramberg K, Christensen K, Jeune B, Skitthe A, Vasegaard L, Vaupel JW. Declining physical abilities with age: a cross-sectional study of older twins and centenarians in Denmark. Age and Ageing 1999; 28: 373-377.

28. Bosh Gil JA, Massagué Ferrer I. Trastornos hematológicos en el anciano. Medicine $7^{a}$ serie. Ed. IDEPSA, Madrid 1999; 5850-54

29. Lipschitz DA. The effect of age on hematopoyesis and the Work-up of anemia in the elderly. Hematology 1999; 504-509.

30. Wieczorowska-Tobis K, Niemir Z, Mossakowska M, Klich-Raczka A, Zyczkowska J. Anemia in centenarians. J Am Geriatr Soc 2002; 50 (7): 1311-3

31. Chan YC, Suzuki M, Yamamoto S. Dietary, anthropometric, hematological and biochemical assessment of the nutritional status of centenarians and elderly people in Okinawa, Japan. J Am Coll Nutr 1997; 16 (3): 229-35. 